\title{
Adenovirus-mediated combined $P 16$ gene and GM-CSF gene therapy for the treatment of established tumor and induction of antitumor immunity
}

\author{
Linhui Wang, Xiaosheng Qi, Yinghao Sun, Li Liang, and Dianwen Ju
}

The Department of Urology, Changhai Hospital, The Second Military Medical University, Shanghai, China.

\begin{abstract}
Antitumor effects of combined transfer of $P 16$ and cytokine genes were investigated in this study. The adenovirus harboring the $P 16$ gene (AdP16) and murine granulocyte-macrophage colony-stimulating factor gene (AdGM-CSF) were utilized for the treatment of established tumors. The mice were inoculated subcutaneously with Renca cells and, 6 days later, received an intratumoral injection of AdP16 in the presence or absence of AdGM-CSF. The results demonstrated that tumor-bearing mice treated with AdP16 in combination with AdGM-CSF showed more potent inhibition of tumor growth and survived much longer than did mice treated with AdP16, AdGM-CSF, adenovirus expressing $\beta$-galactosidase, or phosphate-buffered saline alone $(P<.01)$. The tumor mass showed obvious necrosis and inflammatory cell infiltration, and more $\mathrm{CD}_{4}{ }^{+}$and $\mathrm{CD}_{8}{ }^{+} \mathrm{T}$ cells infiltrating the tumor after combined therapy. After combined therapy, the expression of $\mathrm{MHC}-1\left(\mathrm{H}-2 \mathrm{~K}^{\mathrm{d}}\right)$ and Fas molecules on freshly isolated tumor cells increased greatly. The activity of specific cytotoxic T lymphocytes was also found to be induced more significantly after the combined therapy $(P<.01)$. Our results demonstrated that combined therapy with P16 and GM-CSF genes can inhibit the growth of established tumors in mice significantly and induce antitumor immunity of the host efficiently.
\end{abstract}

Cancer Gene Therapy (2002) 9, 819-824 doi:10.1038/sj.cgt.7700502

Keywords: gene therapy; tumor suppressor gene; adenovirus; renal carcinoma; antitumor immunity; granulocyte-macrophage colony-stimulating factor

G ene therapy, as a promising treatment for many human modalities, encompasses efficient potentials for treating cancers that have failed conventional therapies. The use of P16 gene transfer for the therapy of cancer has been studied extensively. The P16 gene has many features of a tumor suppressor gene. It encodes an inhibitor of cyclin-dependent kinase 4 , which regulates progression through the $\mathrm{G}_{1}$ phase of the cell cycle, ${ }^{1,2}$ and its homozygous deletion is common in tumor-derived cell lines. ${ }^{3,4}$ In view of the fact that adenovirus vectors can deliver genes to a number of tissues at a high rate, we examined adenovirus-mediated $P 16$ gene transfer to murine renal cancer cells (Renca) and evaluated its benefit in treating tumor. The in vitro growth rate of Renca cells after AdP16 treatment was significantly inhibited when compared with the growth rate of AdLacZ - or PBS-treated cells. Our previous studies showed that P16 transduction of Renca cells in vitro could prevent tumor growth in BALB/c mice. Therapy with P16 gene transfection was sometimes effective, but at other times, it was reported to be not efficient in the induction of antitumor immunity, and tumor recurrence or metastasis often occurred after therapy.

Received May 31, 2002.

Address correspondence and reprint requests to: Linhui Wang, MD, The Department of Urology, Changhai Hospital, The Second Military Medical University, Shanghai 200433, China.E-mail:wlhui@online.sh.cn
Direct transfer of cytokine genes into the tumor-bearing host has been demonstrated to be effective for the inhibition of tumor growth. ${ }^{5}$ We have shown that intratumoral injection of recombinant vaccinia virus encoding granulocyte-macrophage colony-stimulating factor (GM-CSF) could exhibit significant antitumor response in melanoma-bearing mice. ${ }^{6}$ Tumor cells transduced with cytokine genes showed increased immunogenicity and decreased tumorigenicity. Tumor vaccine prepared with cytokine gene-modified tumor cells not only induced cellular immunity to protect against the same cells, but also elicited protective immunity to the parental tumor cells. The therapeutic efficacy of one cytokine gene transfection is often not satisfactory especially in pre-established tumors. We have reported that the combined transfer of two cytokine genes, or transfer of one cytokine gene in combination with certain agents, such as interleukin-2 (IL-2) or chemotherapeutic drugs, could induce obvious antitumor immunity of the host. ${ }^{7-9} \mathrm{GM}-$ CSF-secreting tumor cells were found to stimulate potent, specific, and long-lasting antitumor immunity. It was reported that the $G M-C S F$ gene-modified tumor vaccine exhibited the most efficient antitumor efficacy among various cytokine gene-modified vaccines because the locally produced GM-CSF could stimulate the proliferation and differentiation of antigen-presenting cells, thus augmenting the antitumor response of the host.

In this report, we hypothesized that the combined use of P16 gene and GM-CSF gene therapy might have more 
significant antitumor effects than P16 or GM-CSF gene therapy alone, which was proved to be not satisfactory when used alone. To test this hypothesis, we treated tumor-bearing mice with an adenovirus expressing P16 in combination with an adenovirus expressing murine GM-CSF. Inhibition of tumor growth and prolongation of survival period were observed more obviously in tumor-bearing mice after combined therapy when compared with P16 or GM-CSF gene therapy alone. The mechanisms of the antitumor response were also studied in this investigation.

\section{Materials and methods}

\section{Animals and cell lines}

Male or female BALB/c mice, 6-8 weeks of age, purchased from Joint Ventures Sipper BK Experimental Animals (Shanghai, China) were housed for 1 week in a specific pathogen-free state for experiment. Renca, a renal cell carcinoma cell line from BALB/c, and 293, a continuous cell line derived from human embryonic kidney, were maintained in RPMI-1640 medium supplemented with penicillin $100 \mathrm{U} / \mathrm{mL}$, streptomycin $100 \mu \mathrm{g} / \mathrm{mL}, 2$-mercaptoethanol $50 \mathrm{mmol} / \mathrm{L}$, and $10 \%$ fetal calf serum (FCS). All culture media were purchased from Gibco-BRL (Gaithersburg, MD) and FCS provided by the Shanghai Institute of Biological Products (Shanghai, China).

\section{Preparation of recombinant adenoviruses}

Three replication-defective recombinant adenoviruses, AdLacZ (harboring the $\beta$-galactosidase gene), AdP16 (harboring the P16 gene), and AdGM-CSF (harboring the murine $G M-C S F$ gene), were constructed from human adenovirus serotype 5 using homologous recombination. The expression of these genes was driven by a CAG promoter. $\mathrm{CAG}$ promoter is a combination of chicken $\beta$-actin promoter and cytomegalovirus immediate early enhancer. Its function is long-term transcription. This promoter expresses with high efficiency transfected exogenous genes in various mammalian cell lines in vitro. The strong activity of the $\mathrm{CAG}$ promoter has been reported in rodent hepatocytes in vivo. In this study, a plasmid containing a CAG promoter was transfected into an adenovirus and a recombinant adenoviral vector expressing an exogenous gene under the control of the CAG promoter was injected intratumorally into rats, resulting in a high level of transgene expression in the Renca cell.

The recombinant adenoviruses were propagated with 293 cells and the titers of the adenoviral preparations were determined by plaque-forming assay on 293 cells. Briefly, serial 10 -fold dilutions of adenoviruses were added to 24-well plates (Corning, New York, NY) containing confluent 293 cell monolayers. After the 1-hour incubation, the viral dilution media were aspirated and replaced with $2 \mathrm{~mL}$ of agarose overlay; this consisted of equal volumes of $1.2 \%$ Seaplaque agarose (FMC, Rockland, ME) and $\times 2$ DMEM-10\% fetal bovine serum at $37^{\circ} \mathrm{C}$. The agarose overlay was allowed to solidify at room temperature for 20 minutes in a laminar flow hood before the plates were returned to the $37^{\circ} \mathrm{C}$ incubator. Cells were observed for up to 10 days to assess plaque formation. The number of plaques per well was determined, and the plaque-forming units per milliliter of virus inoculum calculated according to the formula:

$$
\mathrm{pfu} / \mathrm{mL}=\frac{\text { average number of plaques per well }}{0.5 \mathrm{~mL} \times \text { viral dilution factor }}
$$

The recombinant adenoviruses produced were diluted to a titer of $10^{9} \mathrm{pfu} / \mathrm{mL}$ in PBS and stored at $-70^{\circ} \mathrm{C}$.

\section{Experimental groups and therapeutic regimens}

Renca cells were taken from continuous culture and resuspended in PBS without FCS for inoculation into mice. BALB/c mice were inoculated subcutaneously (s.c.) with $5 \times 10^{4}$ Renca cells. Six days after tumor inoculation, the tumor-bearing mice were divided into five groups with 15 mice in each group and the mice were injected intratumorally with any of the following preparations: PBS, AdLacZ, AdP16, AdGM-CSF, AdP16 plus AdGM-CSF. Injection of each virus of $10^{8}$ in $0.1 \mathrm{~mL}$ of PBS was performed 6 days after the inoculation of tumor cells and a booster of the same injection was given after 9 days. The length and width of the tumor mass were measured with a caliper every other day after tumor inoculation and the tumor volume was expressed as $0.5 \times$ length $\times$ width $^{2}$.

\section{Histological examination}

S.c. tumor nodules were taken from sacrificed tumorbearing mice 3 days after the last injection. The tumor samples were fixed in $10 \%$ formalin solution, dehydrated, and embedded in paraffin. Thin-sliced sections were stained with hematoxylin and eosin. We used four grades for the pathological analysis of the tumor necrosis: ( - ) no necrosis was present in the tumor; $(+)$ necrosis was present in less than $1 / 3$ of the tumor mass; $(++)$ necrosis was present in $1 / 3-2 / 3$ of the tumor mass; $(+++)$ necrosis was present in more than $2 / 3$ of the tumor mass. For the evaluation of the infiltration of inflammatory cells both in and around the tumor: $(-)$ no inflammatory cell infiltration; $(+)$ minimal inflammatory cell infiltration; $(++)$ medium inflammatory cell infiltration; $(+++)$ intense inflammatory cell infiltration.

\section{Flow cytometry}

S.c. tumors were taken from the sacrificed tumor-bearing mice 3 days after the last injection and the tumor mass was minced. The tumor cells or infiltrated lymphocytes were isolated from the cell suspensions utilizing Ficoll-Hypaque solution. The isolated tumor cells were confirmed to be free of infiltrated immune cells by means of histochemistry. The tumor cells were incubated with optimal concentrations of PE-conjugated monoclonal antibodies against MHC-1 $\left(\mathrm{H}-2 \mathrm{~K}^{\mathrm{d}}\right)$ or Fas for 30 minutes at $4{ }^{\circ} \mathrm{C}$. The tumor cells were washed three times with cold PBS and resuspended in PBS containing $1 \%$ formaldehyde. The lymphocytes were incubated with rat anti-mouse $\mathrm{Ig} \mathrm{CD} 8^{+}$or $\mathrm{CD} 4^{+}$ monoclonal antibodies (PharMingen, San Diego, CA) for 30 minutes followed by three washes with cold PBS. Then 
A

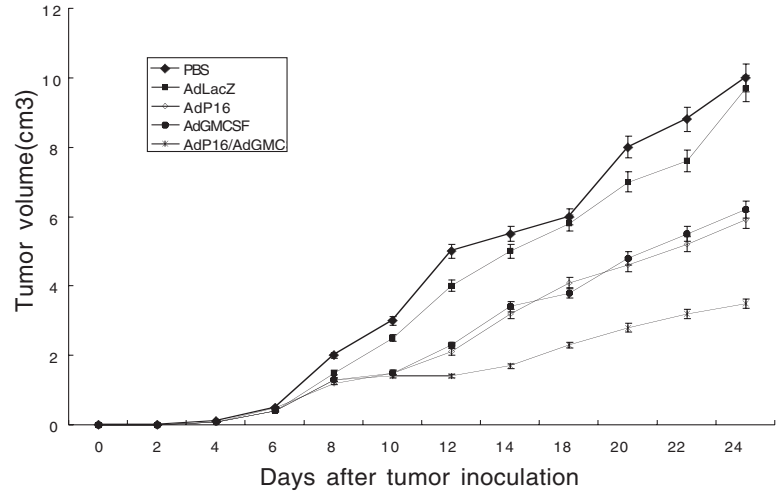

B

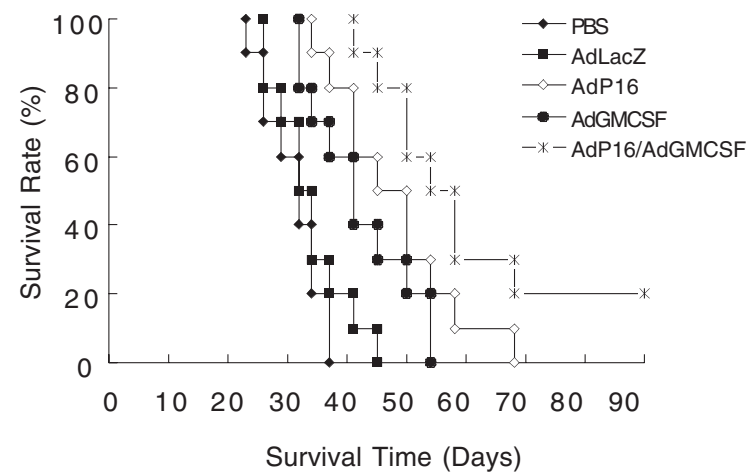

Figure $1 \mathrm{~A}$ : Growth suppression of established Renca s.c. tumors by combined therapy of AdP16 and AdGM-CSF. Tumor volume was expressed as $0.5 \times$ length $\times$ width $^{2}$. B: Survival period of tumorbearing mice after various treatments.

the cells were incubated with fluorescein-isothiocyanateconjugated goat anti-rat IgG for another 30 minutes. The mononuclear cells were then washed with PBS and resuspended in PBS containing 1\% formaldehyde. Control incubations used for determining levels of background staining with the second antibody contained irrelevant normal rat anti-mouse IgG. Flow cytometry results were analyzed on a FACScalibur flow cytometer (Becton Dickinson, San Jose, CA).

\section{Cytotoxic assay}

Splenic lymphocytes were isolated from dead tumor-bearing mice 3 days after the last injection and directly used as NK effector cells. Th lymphocytes were cocultured with inacti- vated Renca cells for 7 days, and then collected as CTL effector cells (CT26). The NK activity and CTL activity were determined by a standard 4 -hour ${ }^{51} \mathrm{Cr}$ release assay. A total of $2 \times 10^{6}$ YAC -1 or Renca cells in $0.5 \mathrm{~mL}$ of RPMI- 1640 with $20 \%$ FCS were labeled with $200 \mu \mathrm{Ci}$ of $\mathrm{Na}{ }^{51} \mathrm{CrO}_{4}$ (Amersham, Arlington Heights, IL) for 2 hours. The labeled cells were washed three times in serum-free medium. A total of $10^{4}$ target cells were then mixed with effector cells for 4 hours at $37^{\circ} \mathrm{C}$ at the ratio indicated. For the maximal ${ }^{51} \mathrm{Cr}$ release control, $0.1 \mathrm{~N} \mathrm{HCl}$ was added to the target cells, and for the spontaneous ${ }^{51} \mathrm{Cr}$ control, $0.1 \mathrm{~mL}$ of medium was added to the labeled cells. The amount of ${ }^{51} \mathrm{Cr}$ released was determined by $\gamma$-counting on a 1275 Minigamma Counter (LKB Wallac, Turku, Finland) and the percentage of specific lysis was calculated as follows:

$\%$ of specific lysis

$$
=\frac{\text { Experimental } \mathrm{cpm}-\text { spontaneous } \mathrm{cpm}}{\text { Maximal } \mathrm{cpm}-\text { spontaneous } \mathrm{cpm}} \times 100
$$

\section{Apoptosis assay of Renca cells}

To determine if apoptosis of tumor cells participated in the process of the antitumor effect, we analyzed the percentages of tumor cells undergoing apoptosis. Renca cells from continuous cultivation in vitro were infected with adenoviruses at a MOI of 20:1 for 24 hours, or Renca cells isolated from dead tumor-bearing mice 3 days after the last injection were stained and apoptosis determined on a Becton Dickinson FACScalibur flow cytometer. Briefly, cells were centrifuged at $1000 \times g$ for 5 minutes, washed with PBS once, and resuspended in $40 \mu \mathrm{L}$ of phosphate-citrate (PC) buffer, consisting of 192 parts of $0.2 \mathrm{M} \mathrm{Na}_{2} \mathrm{HPO}_{4}$ and 8 parts of $0.1 \mathrm{M}$ citric acid ( $\mathrm{pH} 7.8)$, at room temperature for 30 minutes. The samples were then resuspended in $100 \mu \mathrm{L}$ of PBS and $1 \mu \mathrm{L}$ of RNAse A solution $(10 \mathrm{mg} / \mathrm{mL})$, and incubated at $37^{\circ} \mathrm{C}$ for 30 minutes followed by staining with propidium iodide $(50 \mu \mathrm{g} / \mathrm{mL})$ in PBS for 30 minutes. Then the apoptosis rates were determined on a Becton Dickinson FACScalibur flow cytometer.

\section{Statistics}

Statistical analysis was performed using Student's $t$ test. $P<.05$ was considered to be statistically significant.

Table 1 Pathological analysis of tumor mass in tumor-bearing mice after combined therapy with AdP16 and AdGM-CSF

\begin{tabular}{lcccc}
\hline Groups & $\begin{array}{c}\text { Tumor } \\
\text { necrosis }\end{array}$ & $\begin{array}{c}\text { Infiltration of } \\
\text { inflammatory cells } \\
\text { in necrosis area }\end{array}$ & $\begin{array}{c}\text { Infiltration of } \\
\text { inflammatory cells } \\
\text { inside the tumors }\end{array}$ & $\begin{array}{c}\text { Infiltration of } \\
\text { inflammatory cells } \\
\text { around the tumors }\end{array}$ \\
\hline PBS & - & - & - & + \\
AdLacZ & - & - & - & + \\
AdP16 & ++ & ++ & + & ++ \\
AdGM-CSF & ++ & ++ & +++ & ++ \\
AdP16/AdGM-CSF & +++ & ++ & ++ \\
\hline
\end{tabular}

Tumor necrosis: $(+)$ less than $1 / 3$ of the tumor mass; $(++) 1 / 3-2 / 3$ of the tumor mass; $(+++)$ more than $2 / 3$ of the tumor mass. Infiltration of inflammatory cells: $(-)$ no; $(+)$ minimal; $(++)$ medium; $(+++)$ intense. 


\section{Results}

Inhibition of tumor growth and prolongation of survival period in tumor-bearing mice after combined therapy

$\mathrm{BALB} / \mathrm{c}$ mice were inoculated s.c. with $5 \times 10^{4}$ Renca cells and treated with PBS (group A), AdLacZ (group B), AdP16 (group C), AdGM-CSF (group D), or AdP16/AdGM-CSF (group E). The results in Figure 1A demonstrated that inhibition of Renca growth could be observed most significantly in mice 18 days after the treatment with AdP16 in combination with AdGM-CSF (group E) when compared with that in mice treated with control adenovirus AdLacZ, AdP16, or AdGM-CSF therapy alone $(P<.01)$. AdP16 or AdGM-CSF therapy alone also showed a marked inhibitory effect on tumor growth when compared with the PBS control group $(P<.05)$.

Ten tumor-bearing mice in each group were observed for their survival period. The results in Figure 1B demonstrated that the mice that received combined therapy of AdP16/ AdGM-CSF were able to survive much longer than all the other mice that received control therapies $(P<.01)$. Two of 10 mice after AdP16/AdGM-CSF combined therapy were found to be tumor-free and lived for more than 90 days, whereas the control animals after PBS or AdLacZ treatment died within 45 days.

More massive necrosis and inflammatory cell infiltration in tumor mass after combined therapy

We next analyzed the histological changes in tumor sites of mice treated with PBS (group A), AdLacZ (group B), AdP16 (group C), AdGM-CSF (group D), or AdP16/ AdGM-CSF (group E). As shown in Table 1, large areas of tumor necrosis and a great number of inflammatory cells (mainly lymphocytes and neutrophils) were present inside

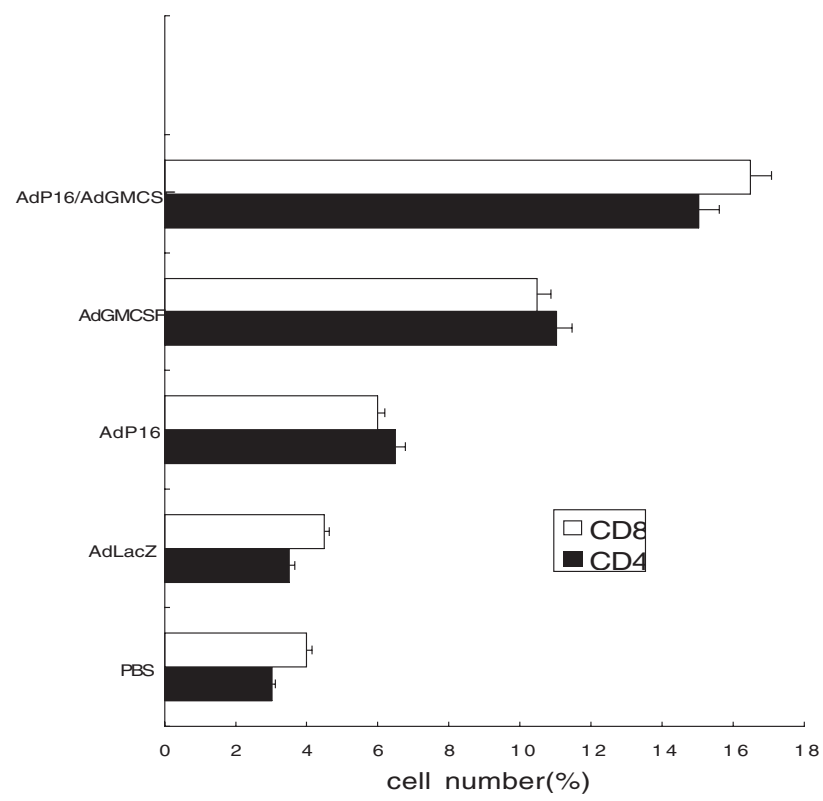

Figure 2 Infiltration of $\mathrm{CD}^{+}, \mathrm{CD} 8^{+} \mathrm{T}$ cells in tumors derived from invarious treated mice. The percentages of $\mathrm{CD}^{+}{ }^{+}$or $\mathrm{CD} 8^{+} \mathrm{T}$ cells in the infiltrating mononuclear cells were determined on a FACScalibur flow cytometer.

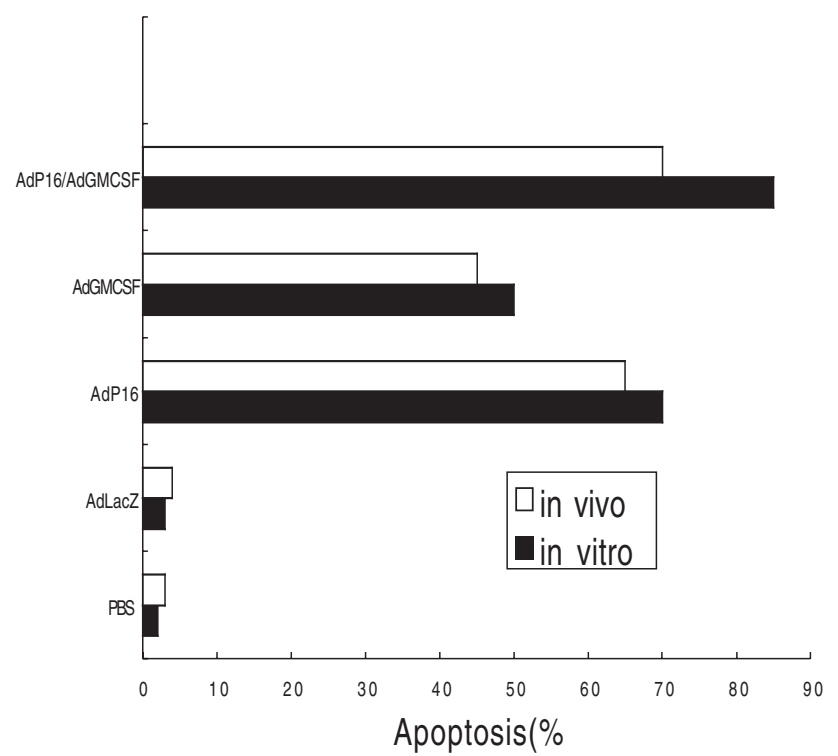

Figure 3 Apoptosis analysis of Renca cells after treatment with AdP16 and/or AdGM-CSF. Renca cells from continuous culture in vitro or freshly isolated from dead tumor-bearing mice were stained and apoptosis determined on a Becton Dickinson FACScalibur flow cytometer.

and around the tumors in mice treated with combined therapy.

More $\mathrm{CD}^{+}, \mathrm{CD}^{+} \mathrm{T}$-cell infiltration in tumor mass after combined therapy

To determine whether local $\mathrm{T}$ cells in a tumor microenvironment were mobilized and activated after combined transfer of P16 and GM-CSF genes, we isolated infiltrating mononuclear cells from a minced tumor mass with Ficoll-Hypaque discontinuous gradient centrifugation. Flow cytometry was performed to measure the $\mathrm{CD}^{+}$and $\mathrm{CD}^{+} \mathrm{T}$ cells in a mononuclear cell suspension. As illustrated in Figure 2, few $\mathrm{CD}^{+}{ }^{+}$and $\mathrm{CD}^{+}{ }^{+} \mathrm{T}$ cells were detected in the mice treated with PBS or AdLacZ. A small number of CD4 ${ }^{+}$and $\mathrm{CD}^{+} \mathrm{T}$ cells were present in AdP16- or AdGM-CSF-treated tumor-bearing mice. More $\mathrm{CD} 4^{+}$and $\mathrm{CD} 8^{+} \mathrm{T}$ cells could be found in AdP16-treated tumor-bearing mice with AdGM-CSF treatment. These data suggest that combined therapy killed the established tumors markedly through transfer of $P 16$ gene, and induced local antitumor immunity of the host efficiently through $G M-C S F$ gene transfer.

Augmentation of immunity and induction of apoptosis in microenvironment of the tumor after combined therapy

MHC class I and Fas molecule expression on the tumor cells after combined therapy with P16 and GM-CSF gene transfers were determined by flow cytometry. Renca cells freshly isolated from PBS- or AdLacZ-treated mice expressed similar levels of MHC-I and Fas when analyzed with PE-conjugated monoclonal antibodies. Treatment of the mice with AdP16 or AdGM-CSF could augment the expression of MHC-I and Fas molecules on the tumor cells significantly when compared with treatment of the mice with PBS or AdLacZ. Treatment of the mice with combined 
therapy could augment the expression of MHC-I and Fas molecules on the tumor cells significantly when compared with treatment of the mice with AdP16 or AdGM-CSF alone.

We used flow cytometry for the determination of the apoptosis of tumor cells both in vitro and in vivo after treatment with PBS, AdLacZ, AdP16, AdGM-CSF, or AdP16/AdGM-CSF. The results in Figure 3 illustrated that neither treatment of the Renca cells with PBS nor with AdLacZ induced apoptosis both in vitro and in vivo. Apoptosis was found in the Renca cells after treatment with AdP16 or AdGM-CSF alone, and obvious apoptosis was found after treatment with AdP16/AdGM-CSF combined therapy.

Induction of more potent specific antitumor immunity in mice after combined therapy with AdP16/AdGM-CSF

Splenocytes from the dead tumor-bearing mice after various therapies were used in cytolytic assays against YAC-1 cells or Renca cells at effector:target $(\mathrm{E}: \mathrm{T})$ ratios of 10:1, 20:1, and $40: 1$ by a 4 -hour ${ }^{51} \mathrm{Cr}$ release assay. The results in Figure 4 demonstrate that AdP16 or AdGM-CSF treatment of the tumor-bearing mice did not enhance splenic NK and CTL activities significantly more than PBS or AdLacZ treatment $(P>.05)$. The combination treatment of AdP16 and
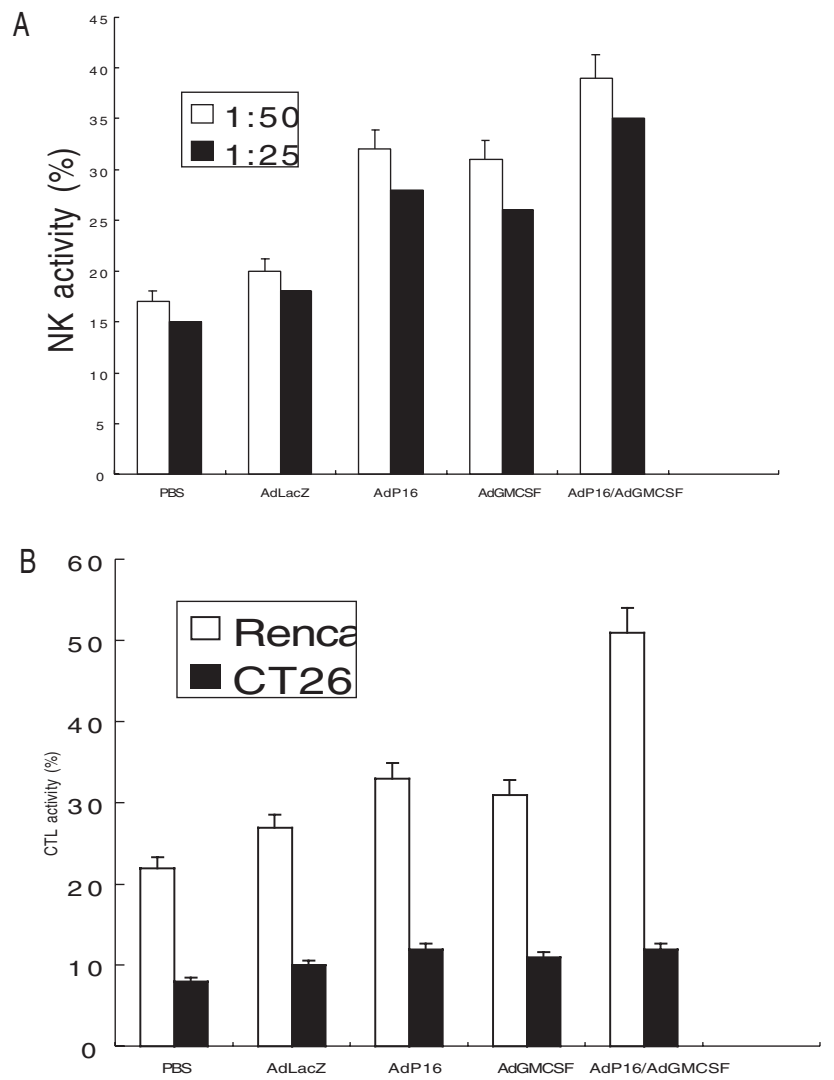

Figure 4 Elevated splenic natural killer (NK, A) and cytotoxic T lymphocyte (CTL, B) activity from tumor-bearing mice after combined therapy with AdP16/AdGM-CSF. Cytotoxicity was determined by a standard 4 -hour ${ }^{51} \mathrm{Cr}$ release assay by utilizing $\mathrm{YAC}-1$ cells and CT26 cells as targets, respectively.
AdGM-CSF increased the NK and CTL activities significantly compared with AdP16, AdGM-CSF, AdLacZ, or PBS $(P<.01)$. No cytotoxicity was found with CTL when syngeneic CT26 cells were used as targets. These data suggest that nonspecific and specific antitumor immunity of the host could be augmented by combined therapy with AdP16 and AdGM-CSF.

\section{Discussion}

Gene therapy of cancer by tumor suppressor gene transfer is an appealing strategy provided that the gene can be efficiently transduced into the tumor cells and the mechanism of function of the particular tumor suppressor can be restored within the tumor. Because tumor cells undergo a number of genetic changes besides loss of function of a particular tumor suppressor, the ultimate goal should not be restoration of function of the lost tumor suppressor, but rather reestablishment of a growth control to an extent which would eventually lead to selective tumor cell death by apoptosis. Our previous studies ${ }^{10}$ showed that in vitro growth rates of Renca cells after AdP16 treatment were significantly inhibited, and cell cycles analysis using flow cytometry showed that AdP16 induced a $\mathrm{G}_{1}-\mathrm{S}$ cycle arrest. In addition, MHC class $\mathrm{I}\left(\mathrm{H}-2 \mathrm{~K}^{\mathrm{d}}\right)$ and costimulatory molecule Fas expression on the P16-transduced Renca cells were also up-regulated.

In this study, antitumor effects were observed in tumorbearing mice after transfer of $P 16$ gene by adenoviral vectors to tumor cells (Renca). But we found that this effect was not satisfactory and the tumor-specific antitumor immunity was not efficiently induced. The NK and CTL activities did not increase significantly more after AdP16 treatment than after PBS or AdLacZ treatment.

GM-CSF is a cytokine with multiple biological effects. The cytotoxic activity of NK cells or CTL can be significantly enhanced or induced by this cytokine. Clinical trials have shown obvious antitumor and immunomodulating effects with GM-CSF gene therapy in renal cell carcinoma, melanoma, lung cancer, bladder cancer, and hematological malignancies. ${ }^{11-13}$ Being a biological response modifier and an efficient immunomodulatory factor in chemotherapy, the antitumor effect of GM-CSF gene therapy alone is usually not strong enough to eradicate established tumors.

To achieve potent antitumor effects and induce antitumor immunity of the host, we simultaneously transduced the P16 and $G M-C S F$ genes into an established tumor. Our results illustrate that the combined therapy suppressed tumor growth and prolonged the survival period of the tumor-bearing mice most significantly. More $\mathrm{CD} 4^{+}$and $\mathrm{CD} 8^{+} \mathrm{T}$ cells infiltrated into the tumors in the mice, and the splenic NK and CTL activities rose significantly in the mice after the combined treatment. The local and systemic antitumor immune responses could clearly be induced after combined transfer of the P16 and GM-CSF genes. The enhanced nonspecific and specific antitumor immunity of the tumor-bearing mice might be responsible for the augmented antitumor effects of combined therapy. In this study, we found obvious infiltration of $\mathrm{CD}^{+}$and $\mathrm{CD} 8^{+} \mathrm{T}$ cells in tumor mass after 
combined treatment. The mechanisms involved in the augmentation of tumor-specific immunity by locally produced GM-CSF are still not clear. The presence of GM-CSF at the site of the tumor may attract $\mathrm{CD}^{+}$and $\mathrm{CD}^{+} \mathrm{T}$ cells from surrounding tissues. These $\mathrm{T}$ cells may play important roles in the processing and presentation of tumor antigens released from AdP16-killed tumor cells, thus enhancing the induction of specific antitumor immune response.

Expression of MHC class I molecule $\left(\mathrm{H}-2 \mathrm{~K}^{\mathrm{d}}\right)$ is very important for the presentation of tumor antigen and the activation of $\mathrm{CD}^{+} \mathrm{T}$ cells. In the present study, we have demonstrated that expression of $\mathrm{H}-2 \mathrm{~K}^{\mathrm{d}}$ on freshly isolated tumor cells increased markedly after P16 gene therapy in combination with GM-CSF gene therapy. We speculated that the tumor cells, with increased MHC-I, may serve as the source of tumor-specific antigen, as well as antigenpresenting cells. In order to investigate if this altered microenvironment of tumor affected the antitumor properties of host lymphocytes, we studied the effector functions of lymphocytes both in the spleen and the tumor mass. Consistent with the above results, we have also demonstrated that more $\mathrm{CD}^{+}$T cells infiltrated into and around the tumor mass after combined gene therapy. Cytotoxicity analysis showed that NK and CTL activities from the spleen in tumor-bearing mice treated with combined therapy increased significantly. The increase in the CTL activity suggested that the antitumor effect of the combined therapy might be through the induction of specific antitumor immunity of the tumor-bearing mice.

Fas (Apo-1/CD95) is a cell surface receptor involved in cell death signaling. Recently, the Fas/Fas ligand system has been identified as a key regulator of apoptosis in some types of cell lines. In this study, we demonstrated that expression of Fas on Renca cells from continuous culture in vitro, or freshly isolated from dead tumor-bearing mice increased markedly after combined therapy. We also found obvious apoptosis of tumor cells both in vitro and in vivo induced by AdP16/AdGM-CSF combined treatment, and the released antigen from the dead tumor cells might be harvested and processed by the GM-CSF-activated antigen-presenting cells. These findings might help to understand the mechanism that more significant tumor cells were killed in vivo after using AdP16/AdGM-CSF combined treatment.

In conclusion, we have show that combined transfer of $P 16$ and GM-CSF genes could inhibit the growth of preestablished renal cell carcinoma and prolong the survival time of the tumor-bearing mice, partially through the efficient induction of specific and nonspecific antitumor immune response. We speculated that the tumor cell debris formed after AdP16/AdGM-CSF combined treatments was processed and presented to the immune system of the host by antigen-presenting cells, e.g., dendritic cells induced by GM-CSF gene transfection. In addition, the tumor cells, which expressed more MHC-I molecules after AdP16/ AdGM-CSF combined therapy, might activate antitumor immune response by acting as antigen-presenting cells themselves. Markedly increased expression of Fas and obvious apoptosis of tumor cells were found in the group that was treated with AdP16/AdGM-CSF combined therapy. The combined therapy, being able to address the drawbacks of cytokine or P16 gene therapy that were proved to be not satisfactory when used alone, might be of great interest for gene therapy of cancer.

\section{References}

1. Serrano M, Gregory J, Beach HD. A new regulatory motif in cell cycle control causing specific inhibition of cyclin D/ CDK4. Nature. 1993;366:704-707.

2. Volker S, Karsten B, Susanne H, et al. Adenovirally transferred P16 INK4/CDKN2 and p53 genes cooperate to induce apoptotic tumor cell death. Nat Med. 1997;3:313-319.

3. Kamb A, Gruis NA, Weaver-feldhaus J, et al. A cell cycle regulator potentially involved in genesis of many tumor types. Science. 1994;264:436-440.

4. Kinoshita H, Yanada H, Ogawa O, et al. Contribution of chromosome 9p21-22 deletion to the progression of human renal cell carcinoma. Jpn J Cancer Res. 1995;86:795-799.

5. Figlin RA, Gitlitz BJ, Belldegrun A. Immunologic approaches to the treatment of cancer. Cancer Invest. 1995;13:339-340. (Editorial).

6. Ju DW, Cao X, Acres B, et al. Intratumoral injection of GMCSF gene-encoded recombinant vaccinia virus elicits potent antitumor response in a mixture melanoma model. Cancer Gene Ther. 1997;4:139-144.

7. Ju DW, Wang BM, Cao X, et al. Adenovirus-mediated combined suicide gene and interleukin-2 gene therapy for the treatment of established tumor and induction of antitumor immunity. J Cancer Res Clin Oncol. 1998;124:683-689.

8. Cao X, Ju DW, Tao Q, et al. Adenovirus-mediated GM-CSF gene and cytosine deaminase gene transfer followed by 5 -fluorocytosine administration elicit more potent antitumor response in tumor bearing mice. Gene Ther. 1998;5:11301136.

9. Cao X, Wang Q, Ju DW, et al. Efficient induction of local and systemic antitumor immune response by liposome-mediated intratumoral co-transfer of interleukin-2 gene and interleukin6 gene. J Exp Clin Cancer Res. 1999;18:191-200.

10. Wang LH, Ju DW, Sun YH, et al. The potent antitumor effects of combined p16 gene and GM-CSF gene therapy through efficient induction of antitumor immunity. J Cancer Res Clin Oncol. 2001;127:101-108.

11. Qin H, Chatterjee SK. Cancer gene therapy using tumor cells infected with recombinant vaccinia virus expressing GM-CSF. Hum Gene Ther. 1996;8:1853-1860.

12. Mahvi DM, Sondel PM, Yang NS, et al. Phase I/IB study of immunization with autologous tumor cells transfected with the GM-CSF gene by particle-mediated transfer in patients with melanoma or sarcoma. Hum Gene Ther. 1997;8:875-891.

13. Shi FS, Weber S, Gan J, et al. Granulocyte-macrophage colony-stimulating factor (GM-CSF) secreted by cDNAtransfected tumor cells induces a more potent antitumor response than exogenous GM-CSF. Cancer Gene Ther. 1999; $6: 81-88$. 